\title{
PROJETO DE DESENVOLVIMENTO SUSTENTÁVEL REGIONAL E LOCAL: UM CASO DE ENSINO
}

\author{
REGIONAL AND LOCAL SUSTAINABLE DEVELOPMENT PROJECT: A TEACHING CASE
}

\author{
Samuel Carvalho de Benedicto \\ Doutor em Administração \\ Pontifícia Universidade Católica de Campinas - PUC-Campinas. \\ Campinas, $\mathrm{SP}-$ Brasil \\ samuel.benedicto@puc-campinas.edu.br
}

Josias Jacintho Bittencourt Pós-Doutor em Direito Universidade de Coimbra - UC Coimbra - Portugal. josias.bittencourt@uol.com.br

Cândido Ferreira da Silva Filho Doutor em Ciências Sociais Pontifícia Universidade Católica de Campinas - PUC-Campinas. Campinas, $\mathrm{SP}-$ Brasil. candidofilho@puc-campinas.edu.br

Cibele Roberta Sugahara Doutora em Ciência da Informação Pontifícia Universidade Católica de Campinas - PUC-Campinas. Campinas, SP - Brasil

cibelesu@puc-campinas.edu.br

\begin{abstract}
Resumo
O presente caso de ensino tem por objetivo aplicar um método didático-pedagógico que permita elaborar um projeto de desenvolvimento sustentável regional e local como solução para os problemas enfrentados por uma Região Integrada de Desenvolvimento (RIDE) brasileira. Neste caso de ensino, foi utilizado o método de ensino denominado PBL (Problem Based Learning), considerado inovador, e uma relevante alternativa ao modelo de ensino tradicional. O caso de ensino é uma situação hipotética que representa a realidade de várias regiões do Brasil. O caso de ensino foi aplicado em uma disciplina do Programa de Pós-Graduação Stricto Sensu em Sustentabilidade de uma universidade da Região Sudeste do Brasil. Utilizando o método PBL, buscou-se nessa disciplina, analisar o problema e propor soluções para o projeto de desenvolvimento sustentável regional e local da RIDE-Plus Ultra. O estudo aponta a necessidade de se estabelecer consórcios municipais, a integração da sociedade local, a formação e capacitação tecnopolítica dos atores sociais e públicos para fundamentar a implementação das ações e obras voltadas ao desenvolvimento sustentável do território. Conclui-se que um pacto social e político, com participação da população em projetos de curto, médio e longo prazo, é fundamental para se alcançar um imprescindível desenvolvimento sustentável regional e local.
\end{abstract}

Palavras-Chave: Projeto de desenvolvimento sustentável. Caso de ensino. Problem based learning. Região integrada de desenvolvimento. Desenvolvimento sustentável regional e local.

\section{Abstract}

This teaching case aims to apply a didactic-pedagogical method that allows the elaboration of a regional and local sustainable development project as a solution to the problems faced by a Brazilian Integrated Development Region (RIDE). In this teaching case, the teaching method called PBL (Problem Based Learning) was used, considered innovative, and a relevant alternative to the traditional teaching model. The teaching case is a hypothetical situation that represents the reality of several regions in Brazil. The teaching case was applied to a discipline of the Stricto Sensu Graduate Program in Sustainability at a university in the southeast region of Brazil. Using the PBL method, this course sought to analyze the problem and propose solutions for the regional and local sustainable development project of RIDE-Plus Ultra. The study points out the need to establish municipal consortiums, the integration of local society, the formation and technopolitical training of social and public actors to support the implementation of actions and works aimed at the sustainable development of the territory. It is concluded that a social and political pact, with the participation of the population in short, medium and long-term projects, are fundamental to achieve an indispensable sustainable regional and local development

Keywords: Sustainable development Project. Teaching case. Problem based learning. Integrated development region. Regional and local sustainable development.

Cite como

American Psychological Association (APA)

Benedicto, S. C., Bittencourt, J. J., Silva Filho, C. F., \& Sugahara, C. R. (2021, maio/ago.). Projeto de desenvolvimento sustentável regional e local: um caso de ensino. Revista de Gestão e Projetos (GeP), 12(2), 163-191. https://doi.org/10.5585/gep.v12i2.19879. 


\section{Introdução}

A preocupação com o desenvolvimento regional sempre esteve presente nas políticas públicas do Brasil. No entanto, o desenvolvimento é o resultado das iniciativas dos indivíduos, empresas e do governo. Em verdade, o sucesso da ação privada e dos indivíduos dependem da ação estatal por meio, por exemplo, de um plano de desenvolvimento e integração regional. Decorre daí a relevância de um projeto de desenvolvimento das Regiões Integradas de Desenvolvimento (RIDE).

Este caso de ensino tem como fundamento o seguinte problema de pesquisa: as RIDE brasileiras compartilham um mesmo desafio, isto é, buscam através das ações econômicas o desenvolvimento sustentável em seu território. No entanto, o desenvolvimento deixou de ser uma simples meta econômica e tem incorporado as dimensões sociais e ambientais, dentre outras. O crescimento econômico é necessário, mas não é suficiente para gerar desenvolvimento sustentável. O conceito de desenvolvimento envolve uma mudança de paradigma, uma vez que contempla o progresso material, qualidade de vida, tecido social e meio ambiente (Lara \& Oliveira, 2017). O "desenvolvimento sustentável tornou-se o conceito central da atual geração" (Sachs, 2015, p. 1). Por sua vez, a sustentabilidade é um conceito difundido ao redor do mundo, uma vez que se refere a um tema de interesse de todos: a manutenção da vida no planeta ao longo do tempo (Nista, Jannuzzi, Falsarella \& De Benedicto, 2020).

$O$ caso de ensino foi desenvolvido utilizando o método de ensino denominado PBL (Problem Based Learning), considerado inovador, e uma relevante alternativa ao modelo de ensino tradicional (Boevé et al., 2017). Diante do contexto exposto, pergunta-se: Como a aplicação do método PBL pode apresentar contribuições para implantar um projeto que visa solucionar os problemas econômicos, sociais e ambientais vivenciados por uma Região Integrada de Desenvolvimento?

Desse modo, o presente caso de ensino tem por objetivo aplicar um método didáticopedagógico que permita elaborar um projeto de desenvolvimento sustentável regional e local como solução para os problemas enfrentados por uma Região Integrada de Desenvolvimento (RIDE) brasileira.

O tópico 2 apresentará aspectos teóricos da metodologia PBL. O tópico 3 e seus subtópicos apresentam o caso de ensino, no qual se utiliza a metodologia PBL para a elaboração de um projeto de desenvolvimento sustentável regional e local.

\section{Metodologia PBL}

O cenário clássico de sala de aula vem sendo contestado internacionalmente, seja pela sua baixa eficiência no processo de construção de 
conhecimento, ou também pela impossibilidade de construir competências que permitam melhorar o trabalho em equipe, a análise crítica, o exercício de pesquisa bibliográfica e documental, entre outras (Castaneda-Ayarza, De Benedicto, Ferreira \& Barbosa, 2019). Os desafios de metodologia de ensino dentro das universidades são muito grandes, tendo em vista que é um ambiente onde predomina a prática de métodos tradicionais.

Diante desse cenário, os educadores carregam a obrigação de sintetizar o vasto volume de informações, especialmente na atual era digital, tecnológica, de inteligência artificial. Somente assim conseguem ajudar os alunos a melhor compreender e colocar em prática o que está disponível no mundo acadêmico (Valente, Almeida, \& Geraldini, 2017).

São diversos os esforços que ocorrem visando inovar os processos de ensino e aprendizagem, que se contraponham aos modelos didáticos de ensino apoiados em perspectivas ditas tradicionais, em que o professor é o centro do processo de transmissão de saberes para alunos que apenas recebem e memorizam o conhecimento transmitido (Souza \& Dourado, 2015).

De acordo com Borochovicius e Tortella (2014), para que a aprendizagem ocorra, ela precisa ser necessariamente transformacional, exigindo do professor uma compreensão de novos significados, relacionando-os às experiências prévias e às vivências dos alunos, permitindo a formulação de problemas que estimulem, desafiem e incentivem novas aprendizagens. Nesse contexto, surge a possibilidade da aplicação do método didático-pedagógico denominado PBL, conhecido também como método de aprendizagem baseado na solução de problemas.

O PBL é uma metodologia ativa que se torna importante por propor o processo de aprendizado centrado no aluno (Silva, Araújo, Leão \& Lins, 2017). O método PBL foi desenvolvido na área de educação médica no Canadá, Holanda e Estados Unidos no começo da década de 1970 e tem sido adotado e adaptado por outras áreas acadêmicas, como administração, arquitetura, direito, engenharia, serviço social e educação (Christopoulos \& Steinbeck, 2016; Leon \& Onófrio, 2015).

Ribeiro (2010) apresenta o PBL como uma estratégia de método para aprendizagem centrada no aluno, que precisa de investigação individual e grupal, que utiliza técnicas de análise crítica para a compreensão e resolução de problemas de forma significativa e em interação contínua com o professor tutor. Segundo Souza e Dourado (2015), no PBL, o objetivo do aluno é solucionar um problema, seja real ou hipotético a partir de um contexto, sendo o protagonista de seu próprio aprendizado através da pesquisa, orientada pelo professor. Assim, o aluno é desafiado a buscar conhecimento, entender e utilizar informações de forma autônoma e reflexiva, para dar respostas aos problemas 
identificados.

Essa metodologia permite aguçar a curiosidade dos alunos e leva à ação de fazer perguntas diante das dúvidas e incertezas sobre os fenômenos complexos do mundo e da vida cotidiana. Nesse processo, os alunos são desafiados a comprometer-se na busca pelo conhecimento, por meio de questionamentos e investigação, para dar respostas aos problemas identificados (Tainter, Wong, Cudemus-Deseda \& Bittner, 2017).

Segundo Castaneda-Ayarza et al. (2019), diferentemente do método tradicional, os métodos ativos propõem que o processo esteja centrado no estudante, estimulando-o a buscar, entender e utilizar informações de forma autônoma e reflexiva, auxiliando na discussão e na proposta de soluções junto a seus pares. Desse modo, na metodologia PBL o processo de aprendizagem é sempre ativo e colaborativo, ou seja, envolve não apenas uma ação pessoal do indivíduo, mas também trabalhos e atividades acadêmicas em equipe, em grupo.

$\mathrm{O}$ desafio do PBL é transformar o estudante em um ser independente e autônomo no processo de aprendizagem (Frezatti, Martins, Mucci \& Lopes, 2018). Comparativamente ao método tradicional, o PBL oferece diversas vantagens, como o desenvolvimento da autonomia, a interdisciplinaridade, a indissociabilidade entre teoria e prática, o desenvolvimento do raciocínio crítico e de habilidades de comunicação e a educação permanente (Borges, Chachá, Quintana, Freitas \& Rodrigues, 2014).

Para Nagarajan e Overton (2019), o método PBL demonstra ser bastante relevante diante dos métodos de ensino tradicionais, conservadores. Nesse sentido, Gholami et al. (2016) destacam que a abordagem PBL pode ajudar a desenvolver a consciência metacognitiva e melhorar as habilidades de pensamento crítico de estudantes, sem qualquer prejuízo nos testes de conhecimento em comparação com os alunos da aula expositiva.

Ao tratar dessa temática, Sugahara, Jannuzzi e Sousa (2012) afirmam que o processo de aprendizagem com abordagem do PBL requer a renovação das concepções do que é ser professor. Todavia, a inovação pedagógica do processo de ensino e aprendizagem não ocorrerá espontaneamente, nem de um momento para outro, em função da característica inerente da abordagem do PBL, sobretudo, no que concerne à uma prática docente participativa que possibilite estudos e simulações do conteúdo teóricoconceitual com a prática desenvolvida no mundo do trabalho.

Do ponto de vista prático, Ribeiro e Mizukami (2005) apontam que o método PBL traz mudanças para o papel dos professores e estudantes. Cria-se um grupo tutorial, composto de um tutor (professor) e de cinco a oito estudantes, dentre os quais haverá um líder, um redator e um porta voz. Os professores passam a ser tutores com a função de orientar, facilitar, 
explicar conceitos, ajudar os alunos a delinear questões, sanar dúvidas com relação aos requisitos do projeto e às tarefas a serem cumpridas. Um bom tutor, segundo Araújo, Fregonesi, Soares e Slomski (2010), deve ter as seguintes características: conhecimento, atributos pessoais (aceitação e responsabilidades) e habilidades relacionais.

No método PBL, o problema, baseado em situações significativas e contextualizadas no mundo real, é o ponto de partida para o processo de aprendizagem (Escrivão Filho \& Ribeiro, 2008). Ylitalo, Jääskeläinen, Horelli e Väänänen (2012) ressaltam a importância de utilizar casos reais ou que retratam hipoteticamente uma realidade para o processo de aprendizagem no método PBL. Dessa forma facilita-se e melhorase a eficiência das atividades laboratoriais e de pesquisa bibliográfica e documental, as quais serão fundamentais para a construção dos novos conhecimentos e da proposta de solução do problema proposto.

$\mathrm{O}$ problema precede à teoria, atuando como o foco da aprendizagem, promovendo a integração dos conceitos e habilidades necessários para sua solução. Na sequência, disponibilizam-se aos estudantes recursos e orientações à medida que se desenvolve a solução do problema proposto e a evolução dos conteúdos da disciplina (Freitas, 2012). A Figura 1 apresenta uma sequência circular e dinâmica das atividades envolvidas no método PBL.

Figura 1 - Sequência das atividades envolvidas no método PBL

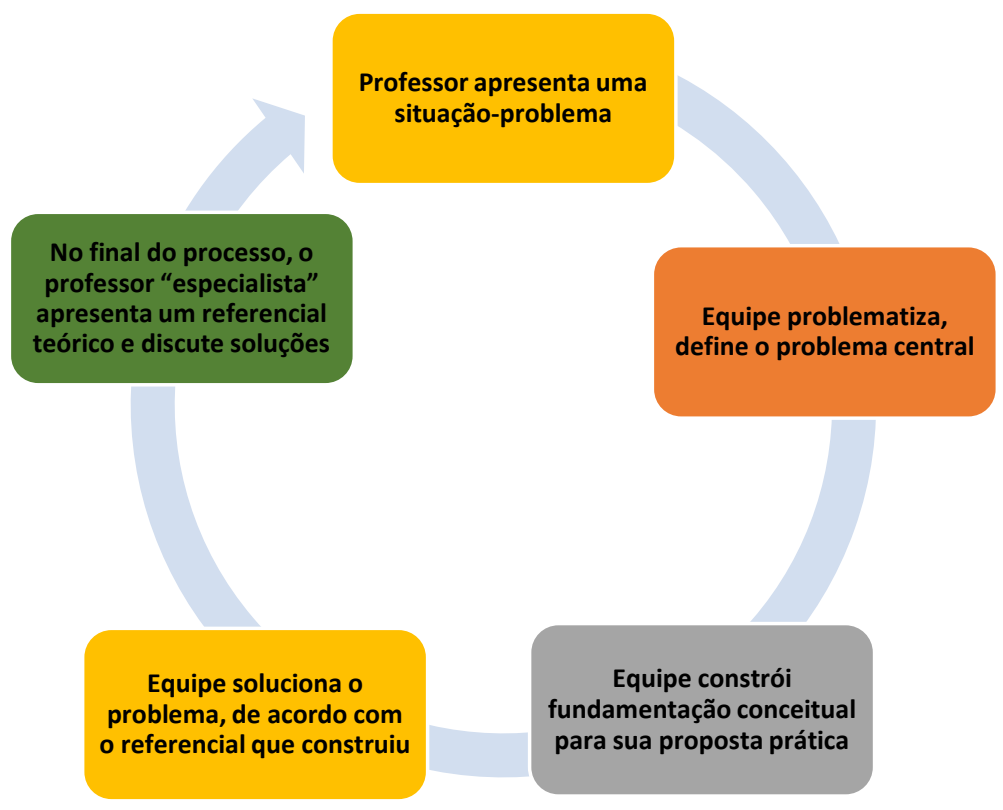

Fonte: Carvalho (2018).

Do ponto de vista operacional, ao trabalhar com a metodologia PBL o professor, 
geralmente, segue as etapas abaixo:

Formação de equipe - Inicialmente o professor cria uma equipe composta por um tutor (professor) e cinco a oito estudantes. Os estudantes são assim divididos: um coordenador, um redator, um porta voz e os demais são membros regulares. Podem ser formadas várias equipes, a depender do número total de alunos na classe.

\section{Apresentação do problema - $\mathrm{O}$} professor apresenta aos alunos um problema real ou hipotético (simulado). Um problema hipotético precisa se aproximar bem da realidade. O problema, seja real ou hipotético, precisa ser amplo (não minucioso) a fim de aguçar a imaginação dos alunos.

Relatório parcial - Após receber o problema, os alunos elaboram um relatório parcial sobre o tema que estão estudando. Este relatório é básico, pois é o início das atividades que os alunos desenvolverão para que possam solucionar o problema que foi apresentado. O relatório parcial deve conter: (i) definição dos fatos encontrados no problema; (ii) definição das causas do problema; (iii) definição da síntese do problema a partir dos fatos e causas detectados; (iv) definição dos conceitos teóricos que serão estudados para fundamentar a elaboração de um relatório final; (v) divisão das tarefas e datas das reuniões da equipe.

Pesquisa teórica - Após definir os conceitos a serem pesquisados, os alunos iniciam o processo de pesquisa teórica. Cada aluno é responsável por um ou mais tópicos de pesquisa. Após a pesquisa individual, a equipe se reúne quantas vezes forem necessárias para a elaboração do relatório final.

Relatório final - Este relatório contém a essência de tudo o que foi pesquisado pelos alunos e deve ser entregue ao professor da disciplina para que seja avaliado. O relatório final é composto pelas seguintes fases: (i) uma introdução, com informações básicas sobre o problema e as estratégias de pesquisa; (ii) os conceitos relevantes e a teoria utilizada; (iii) e as soluções, nas quais os alunos sugerem ações de curto, médio e longo prazo para o problema estudado.

Apresentação - Nessa etapa, os alunos apresentam para os colegas e o professor os conceitos pesquisados e as soluções que encontraram para o problema.

Debate - Após a apresentação, ocorre o debate entre as equipes da sala. Nesse momento, os grupos fazem perguntas uns para os outros para verificar o que entenderam das apresentações dos outros grupos e sobre os conteúdos que estão sendo ensinados.

Fundamentação teórica - Essa é a fase final de um PBL, na qual o professor faz uma aula teórica e dialogada para o fechamento da situação problema estudada. Nesse momento, o professor explica os tópicos estudados pelos alunos e também explora algum tópico que deveria ter sido estudado, mas que, algumas vezes, deixa de ser abordado pelos alunos. Este é o momento 
apropriado para os alunos tirarem suas dúvidas.

Após expor aspectos teóricos do método PBL, serão apresentadas nos próximos tópicos as etapas vistas anteriormente, mas agora contemplando aspectos práticos do caso de ensino envolvendo a elaboração de um projeto de desenvolvimento sustentável regional e local visando a solução dos problemas enfrentados por uma Região Integrada de Desenvolvimento (RIDE).

\section{Caso de ensino - Etapas do PBL}

Neste tópico descreve-se o caso de ensino, o qual foi construído em etapas conforme orientado pela metodologia PBL. Este caso de ensino foi organizado nas seguintes etapas: formação da equipe; apresentação do problema; elaboração do relatório parcial; elaboração do relatório final; apresentação e debate sobre o relatório final; e, finalmente, a fundamentação teórica feita pelo professor.

\subsection{Formação de equipe}

Devido ao número limitado de alunos na classe, o professor decidiu criar apenas uma equipe de trabalho, composta por oito estudantes. Os estudantes foram assim distribuídos na equipe: um coordenador geral (líder), um redator, um porta-voz e os demais deviam atuar como membros regulares.

\subsection{Apresentação do problema}

O Brasil adota o Federalismo como seu sistema de Governo. O Federalismo, segundo Fortes e Moraes (2016), é o modelo mais adequado para um país vasto como o Brasil, com suas desigualdades tanto econômicas quanto culturais e territoriais. Com a Constituição Federal de 1988, que fortaleceu a figura do Município na Federação brasileira, estes deixam de ser entes tutelados pelos Estados e passam a figurar como ente federativo, passando a ser responsáveis por um grande leque de atividades de governo. A descentralização política do estado brasileiro transferiu diversos serviços públicos aos municípios em contraponto à contínua concentração dos recursos públicos no nível central (governo federal), agravando as desigualdades regionais e a baixa capacidade técnica das gestões municipais. Diante desta realidade, muitos problemas se afloram nos municípios Brasil à fora.

Plus Ultra é uma Cidade Polo, com 600 mil habitantes, cercada por outras 14 cidades de menor porte. Plus Ultra (e suas cidades satélites) está localizada numa região geográfica pouco desenvolvida socioeconomicamente, denominada Região Integrada de Desenvolvimento (RIDE). Há um movimento integrado entre os prefeitos da RIDE-Plus Ultra no sentido de promover o desenvolvimento sustentável da região e de cada cidade individualmente.

Os prefeitos das 15 cidades da RIDE-Plus Ultra encomendaram um estudo para levantar os 
principais problemas enfrentados pelos respectivos municípios. Os resultados do estudo apontaram os seguintes problemas: falta de vagas em abrigos para crianças e adolescentes; necessidade de ampliar a rede escolar para atender a demanda de crianças e adolescentes; poucas opções de cursos profissionalizantes para jovens; poucas opções de cursos superiores; baixa informatização das escolas públicas; baixo acesso e incentivo à cultura; defesa civil despreparada para atender as necessidades da população em momentos de risco; iluminação pública deficitária; falta de qualificação de muitos servidores que atuam diretamente na gestão pública; necessidade de melhoria na segurança pública; mobilidade urbana comprometida; necessidade de integrar o sistema de transporte público; gestão patrimonial deficitária; máquinas e equipamentos sucateados; falta de recursos financeiros para a compra de tratores, maquinas e equipamentos para atender a zona rural; necessidade de ampliar a assistência técnica aos produtores rurais; necessidade de criação de política de uso sustentável do solo; estradas que interligam os municípios em condições precárias; jogo de empurra entre os municípios quanto ao atendimento à população em locais limítrofes; déficit no abastecimento de água; falta de saneamento em bairros periféricos; falta de projetos ambientais; necessidade de proteção de bacias hidrográficas; melhor aproveitamento das opções turísticas; necessidade de criação de aterro sanitário para o tratamento de resíduos sólidos; sobrecarga dos postos de saúde e pequenos hospitais; necessidade de construção de um hospital de grande porte; necessidade de ampliação do corpo médico com especialidades diferentes; alto custo na aquisição de medicamentos feito por cada município isoladamente; alto custo na aquisição de merenda escolar feito por cada município isoladamente; alto custo na aquisição de insumos gerais feito por cada município isoladamente; necessidade de atração de empresas para a geração de novos empregos; dentre outros.

O estudo realizado pelos prefeitos revelou que, por um lado, boa parte dos problemas e desafios encontrados não se limitam ao território de um município isoladamente. Diversos municípios são assolados por problemas e desafios comuns. Por outro lado, tais problemas e desafios, não chegam a impactar o âmbito estadual. Diante desta realidade, os prefeitos passaram a indagar: Quais estratégias administrativas são fundamentais para a solução de grandes problemas enfrentados por uma Região Integrada de Desenvolvimento (RIDE), cujos resultados não fiquem restritos a um município isoladamente? Quais opções temos para promover o desenvolvimento das cidades que compõem a RIDE-Plus Ultra de tal forma que seus entes federados sejam beneficiados coletivamente? Como agem os municípios em outros países quando enfrentam esses mesmos problemas e desafios? 
Depois de muitos palpites..., finalmente, os prefeitos das 15 cidades da RIDE-Plus Ultra decidiram buscar a ajuda do Programa de PósGraduação Stricto Sensu em Sustentabilidade de uma Universidade do Sudeste brasileiro. O professor da disciplina de "Estratégias Públicas e Privadas de Desenvolvimento Local e Metropolitano" submeteu o problema da RIDEPlus Ultra aos alunos. O desafio posto devia envolver a elaboração de um projeto de desenvolvimento sustentável regional e local (DSRL)para a RIDE-Plus Ultra utilizando a metodologia PBL.

\subsection{Relatório parcial}

Com o problema em mãos, os alunos começaram a elaborar o relatório parcial. O relatório parcial foi estruturado da seguinte forma:

\section{1) Fatos encontrados no problema:}

- Há um movimento integrado entre os prefeitos da RIDE-Plus Ultra;

- Há necessidade de integrar o sistema de transporte público;

- Há um jogo de empurra entre os municípios quanto ao atendimento à população em locais limítrofes;

- Há necessidade de proteção de bacias hidrográficas;

- Necessita-se de um melhor aproveitamento das opções turísticas;
- Necessidade de criação de aterro sanitário para o tratamento de resíduos sólidos;

- Há sobrecarga dos postos de saúde e pequenos hospitais;

- É necessário construir um hospital de grande porte;

- $O$ custo na aquisição de medicamentos feito por cada município isoladamente é alto;

- O custo na aquisição de merenda escolar feito por cada município isoladamente é alto;

- O custo na aquisição de insumos gerais feito por cada município isoladamente é alto;

- Boa parte dos problemas e desafios encontrados não se limitam ao território de um município isoladamente;

- Diversos municípios são assolados por problemas e desafios comuns;

- Os problemas e desafios não chegam a impactar o âmbito estadual;

- Há dúvidas sobre quais estratégias administrativas são fundamentais para a solução de grandes problemas enfrentados..., cujos resultados não fiquem restritos a um município isoladamente;

- Há dúvidas sobre quais opções para promover o desenvolvimento das cidades que compõem a RIDE-Plus Ultra de tal forma que seus entes federados sejam beneficiados coletivamente. 
- Há dúvidas sobre como agem os municípios em outros países quando enfrentam esses mesmos problemas e desafios.

\section{2) Causas do problema:}

- Agravamento da desigualdade na região devido ao sistema federativo e questões políticas históricas;

- Baixa capacidade técnica das gestões municipais da RIDE-Plus Ultra ocasionada, em parte, pela falta de qualificação dos servidores públicos;

- Falta de um planejamento integrado envolvendo toda a RIDE-Plus Ultra, levando em consideração as especificidades e potencialidades de cada cidade;

- Falta de projetos bem fundamentados para a captação de recurso juntos as esferas estadual e federal;

- Falta de integração regional de alguns serviços públicos como: sistema de transporte, proteção de bacias hidrográficas, promoção do turismo, destino e tratamento de resíduos, atendimento médico-hospitalar, compras de produtos de alto custo, etc.;

- Falta de políticas de integração entre o espaço rural e o urbano;

- Falta de políticas de proteção do meio ambiente;
- Falta de envolvimento dos pequenos e grandes empresários no processo de desenvolvimento socioeconômico da RIDE-Plus Ultra;

- Ausência de Arranjos Produtivos Locais (APLs) para aproveitar as vocações de cada cidade;

- Ausência de incubadoras de empresas e de um parque tecnológico;

- Dentre outras.

\section{3) Síntese do problema a partir dos fatos e causas detectados:}

O problema vivenciado pela RIDE-Plus Ultra foi sintetizado da seguinte forma: "A intenção de promover um desenvolvimento sustentável regional e local somente poderá se concretizar com um planejamento de curto, médio e longo prazo".

\section{4) Conceitos teóricos a serem estudados para fundamentar a elaboração do relatório final:}

Após identificar os fatos, as causas e elaborar uma síntese do problema, os alunos realizaram uma consulta a especialistas na área de políticas públicas para desenvolvimento regional e local e um prévio estudo de campo. Com base nesses elementos, os alunos definiram os conceitos teóricos que deveriam ser pesquisados para elaborar um projeto de desenvolvimento da RIDE-Plus Ultra. Os alunos realizaram buscas no Portal de Periódicos Capes, Portal do Sebrae, 
Portal da Scielo, Google Acadêmico, Biblioteca

Digital Brasileira de Teses e Dissertações (BDTD) e Catálogo de Teses e Dissertações da Capes. Os termos buscados foram: desenvolvimento sustentável, sustentabilidade, desenvolvimento sustentável regional e local, desenvolvimento metropolitano, Região Integrada de Desenvolvimento (RIDE), projeto de desenvolvimento sustentável. Após algumas leituras prévias, os alunos definiram os tópicos de pesquisa, quais sejam:

(i) desenvolvimento sustentável e sustentabilidade;

(ii) projeto de desenvolvimento sustentável regional e local;

(iii) governança metropolitana sustentável; (iv) integração social, econômica e ambiental entre municípios para o desenvolvimento sustentável;

(v) cooperativismo e consórcio intermunicipal sustentável;

(vi) políticas públicas para o desenvolvimento local e consórcio intermunicipal sustentável;

(vii) aglomerações produtivas, clusters, APLs e incubadoras.

\section{5) Divisão das tarefas e datas das reuniões da equipe:}

As tarefas de pesquisa para a elaboração do relatório final foram divididas entre os alunos de acordo com o Quadro 1.

Quadro 1 - Tarefas de pesquisa de cada aluno e respectiva data de entrega

\begin{tabular}{|l|l|l|}
\hline Aluno & Tópico de pesquisa & Data da entrega \\
\hline A & (i) desenvolvimento sustentável e sustentabilidade & $\ldots \ldots . . \ldots \ldots / \ldots \ldots$ \\
\hline B & (ii) projeto de desenvolvimento sustentável regional e local & $\ldots \ldots . . \ldots \ldots / \ldots \ldots$ \\
\hline C & (iii) governança metropolitana sustentável & $\ldots \ldots . . \ldots \ldots / \ldots \ldots$ \\
\hline E & $\begin{array}{l}\text { (iv) integração social, econômica e ambiental entre municípios para o } \\
\text { desenvolvimento sustentável }\end{array}$ & $\ldots \ldots . . \ldots \ldots / \ldots \ldots$ \\
\hline F & (v) cooperativismo e consórcio intermunicipal sustentável & $\ldots \ldots . / \ldots \ldots / \ldots \ldots$ \\
\hline G & $\begin{array}{l}\text { (vi) políticas públicas para o desenvolvimento local e consórcio } \\
\text { intermunicipal sustentável }\end{array}$ & $\ldots \ldots . / \ldots \ldots . \ldots \ldots$ \\
\hline
\end{tabular}

Fonte: Equipe do PBL.

Foram agendadas cinco reuniões da equipe para definir as soluções e ações de curto, médio e longo prazo e a elaboração do relatório final.

\subsection{Relatório final}

Após a realização da pesquisa teórica sobre os tópicos mencionados, os alunos se reuniram e elaboraram um relatório final, o qual foi entregue ao professor para ser avaliado. $\mathrm{O}$ relatório final, apresentado abaixo, foi composto de três etapas: (i) a introdução, com informações básicas sobre o problema e as estratégias de pesquisa; (ii) os conceitos relevantes pesquisados; 
(iii) as soluções, com ações de curto, médio e longo prazo propostas pelos alunos.

\subsubsection{Introdução}

Tomando como ponto de partida os problemas apresentados pelos Prefeitos da RIDEPlus Ultra, os alunos fizeram uso da metodologia PBL e apresentaram um relatório que seria a base para o projeto de DSRL dirigido à RIDE-Plus Ultra.

O estudo partiu do seguinte problema hipotético: Com o fortalecimento dos municípios brasileiros como entes federativos, pela Constituição de 1988, estes passaram a ser responsáveis por várias atividades do governo. Essa descentralização política acabou transferindo diversos serviços públicos diretamente aos municípios, o que acabou agravando as desigualdades regionais e a baixa capacidade técnica das gestões municipais (Carvalho, 2007). Este estudo se torna importante devido as Regiões Integradas de Desenvolvimento (RIDE) brasileiras compartilharem um mesmo desafio: a busca pelo desenvolvimento sustentável em seus territórios, geralmente, por meio de ações puramente econômicas (Matus, 2000). Com este cenário base, foi criado o PBL, onde Plus Ultra é caracterizada como uma Cidade Polo, com 600 mil habitantes, cercada por outras 14 cidades de menor porte, que estão localizadas em uma região pouco desenvolvida, denominada RIDE-Plus
Ultra.

Os 15 prefeitos das cidades que compõem a RIDE-Plus Ultra entendem que a região possui problemas em comum, com impactos ambientais, sociais e econômicos negativos, não se limitando ao território de um município isoladamente. Assim, os prefeitos tiveram a iniciativa de procurar estratégias administrativas para a solução dos grandes problemas enfrentados na RIDE-Plus Ultra e opções para promover o desenvolvimento das cidades.

Para este comprometimento ser completo, há a necessidade de um salto qualitativo e quantitativo quanto aos aspectos econômico, social, ambiental. Os responsáveis pela mudança devem pesquisar sobre políticas e linhas de crédito para implementar o projeto e pessoas preparadas para lidar com este novo desafio. Estudar políticas públicas voltadas para o desenvolvimento local sob uma perspectiva sustentável é muito importante para a região. A RIDE-Plus Ultra deve ainda analisar as vocações econômicas que precisam ser valorizadas e aproveitadas e o envolvimento dos empresários da região. Deve também considerar a criação de mecanismos para melhor organizar os empresários e criar uma estrutura para abrigar as novas empresas. Considerar os empreendimentos sociais, que envolvam as comunidades carentes, e os empreendimentos de natureza social também devem ser considerados.

Uma vez que este cenário de cidades com desafios comuns, em áreas menos favorecidas 
economicamente e de interesse do Estado são realidade do país, e em grande número, este estudo pode ser uma referência básica para interessados em evoluir como RIDE ou Consórcios Intermunicipais, por apresentar um estudo utilizando a metodologia PBL, onde os resultados são apresentados em cenários de curto, médio e longo prazo. Com base na pesquisa bibliográfica realizada pelos alunos, numa consulta feita a especialistas na área de políticas públicas para desenvolvimento regional e local e num estudo de campo, foram criadas algumas categorias de análise, as quais serão apresentadas e discutidas a seguir.

\subsubsection{Desenvolvimento sustentável e sustentabilidade}

Sustentável, sustentabilidade e desenvolvimento sustentável são termos notáveis e oportunos em nível global. Embora esses termos sejam muito utilizados na literatura científica, ainda não existe consenso sobre um conceito para os mesmos. Desse modo, "na literatura, existe uma vasta diversidade de conceitos, relacionada, de forma predominante, com o desenvolvimento sustentável" (Feil \& Schreiber, 2017, p. 668).

$$
\mathrm{O} \text { conceito mais difundido de }
$$
desenvolvimento sustentável se encontra no Relatório Brundtland "Nosso Futuro Comum" (Japiassú \& Guerra, 2017). De acordo com esse documento, o desenvolvimento sustentável pode ser compreendido como "aquele que atende às necessidades do presente sem comprometer a possibilidade de as gerações futuras atenderem a suas próprias necessidades" (Goldenberg, 2015, p. 33).

De acordo com Nascimento (2012), sustentabilidade é a capacidade de um sistema humano, natural ou misto resistir ou se adaptar à mudança endógena ou exógena por tempo indeterminado. Por outro lado, o desenvolvimento sustentável é uma via de mudança intencional e melhoria que mantém ou aumenta esse atributo do sistema, ao responder às necessidades da população presente. Sendo assim, o desenvolvimento sustentável é o caminho para se alcançar a sustentabilidade, caracterizando-a como objetivo, e não meio.

Apesar de entender que sustentabilidade e desenvolvimento sustentável não são sinônimos, neste estudo os mesmos serão tratados como termos equivalentes, conforme Sartori, Latrônico e Campos (2014) defendem essa possibilidade.

O desenvolvimento sustentável implica na maximização simultânea de objetivos do sistema biológico (como a diversidade genética, resiliência e produtividade biológica), objetivos do sistema econômico (caracterizadas pela satisfação das necessidades básicas, aprimoramento da equidade e o incremento no uso de bens e serviços), e objetivos do sistema social (intrínsecos à diversidade cultural, sustentabilidade institucional, justiça social e participação). (Pott \& Estrela, 2017). A busca pelo desenvolvimento sustentável é 
multifacetada, não podendo depender de um único fator. Assim, nos últimos anos, o desenvolvimento sustentável passou a ser visto como um conceito multidimensional que prioriza, não somente aspectos de crescimento econômico, mas também de questões ambientais, sociais, territoriais, dentre outras (Oliveira, 2018).

De fato, o conceito de desenvolvimento sustentável extrapolou as crises ambientais, "adentrando no contexto das mais recentes crises sociais e econômicas, como a pobreza, problemas de saúde pública e o alargamento das distâncias entre economias industrializadas e economias emergentes" (Machado \& Matos, 2020, p. 22). Desse modo, os problemas que afetam o desenvolvimento de um local ou região não são apenas econômicos, como querem alguns. São problemas econômicos, sociais, ambientais, políticos, territoriais, culturais e tecnológicos, os quais se enquadram nas dimensões da sustentabilidade. Desse modo, o desenvolvimento regional e local de uma Região Integrada de Desenvolvimento encontra guarida nos conceitos de desenvolvimento sustentável e sustentabilidade (ENAP, 2018).

\subsubsection{Projeto de desenvolvimento sustentável $\underline{\text { regional e local }}$}

O desenvolvimento sustentável regional e local (DSRL) é o processo de aproveitamento das vantagens comparativas e competitivas de uma localidade para favorecer o seu crescimento econômico, melhorar a qualidade de vida da população, fortalecer o capital social, promover uma boa governança e o uso sustentável dos recursos naturais. Nota-se que para um desenvolvimento regional e local convergem fatores ambientais, sociais, econômicos, políticos e institucionais, que se cruzam e se interpenetram (Paula, 2008).

O DSRL tem por objetivo melhorar a qualidade de vida das pessoas, bem como o aumento gradativo do acesso de todos a um território (campo e cidade) mais digno e justo de se viver, mantendo, do mesmo modo, a preocupação com o meio ambiente e a garantia de sua regeneração natural. Envolve a possibilidade de aplicar, de maneira eficaz, a riqueza gerada pela localidade, em virtude do seu crescimento econômico, visando equilíbrio social e ambiental (Albarello, Albarello \& Siedenberg, 2006).

Segundo Marques, Barazzutti, Senna e Alves (2014), para que o desenvolvimento regional e local seja sustentável, é necessária a valorização das potencialidades locais existentes, de tal maneira que possibilite oportunidades sociais para viabilizar e contribuir com o desenvolvimento da economia local.

Para Arenti (2008, p. 22), as políticas públicas voltadas para o desenvolvimento sustentável de uma nação devem "levar em consideração as vantagens comparativas regionais, a formação de clusters locais e de sistemas locais de inovações". O pressuposto é que as esferas mais descentralizadas de poder têm maior conhecimento e maior capacidade de 
operação para implementar políticas de desenvolvimento sustentável. Ao tratar dessa temática, Albagli (2006) afirma que os espaços locais e regionais ganham relevância na estratégia de desenvolvimento sustentável ao exigir a participação das pessoas que ali se encontram por possuírem conhecimentos e serem culturalmente e socialmente pertencentes à região onde vivem, possibilitando que o desenvolvimento ocorra de forma endógena.

O DSRL é uma estratégia negocial que busca impulsionar o desenvolvimento sustentável das regiões brasileiras. Segundo Lima (2006), o DSRL propõe-se a:

(i) promover geração de trabalho e renda e a inclusão social;

(ii) inserir as pessoas no mercado consumidor;

(iii) democratizar o acesso ao crédito;

(iv) disseminar a cultura empreendedora;

(v) desenvolver capitais humano, produtivo e social;

(vi) incentivar o associativismo e o cooperativismo;

(vii) contribuir para melhoria dos indicadores de qualidade de vida;

(viii) preservar o meio ambiente;

(ix) dentre outras ações.

Lima (2006) enfatiza que DSRL baseiase nos conceitos de desenvolvimento e sustentabilidade. $\mathrm{O}$ desenvolvimento é resultante da presença dos seguintes fatores: (i) capital humano (conhecimentos, habilidades, competências);

(ii) capital social (confiança, cooperação, organização);

(iii) capital produtivo (empreendimentos, serviços e tecnologia);

(iv) cultura empreendedora (iniciativa e atitude proativa).

Já a sustentabilidade é resultante do equilíbrio entre as dimensões propostas por Sachs (2009), as quais devem ser:

(i) economicamente viável;

(ii) socialmente justa;

(iii) ambientalmente correta;

(iv) culturalmente diversa;

(v) territorialmente planejada e bem distribuída;

(vi) politicamente dirigida ao bem comum;

(vii) tecnologicamente útil;

(viii) educacionalmente formadora $\mathrm{e}$ transformadora.

No que diz respeito ao desenvolvimento sustentável e sustentabilidade na gestão de projetos, Cavalcanti e Silva (2016) mencionam que sua inclusão no gerenciamento de projetos ainda é superficial, tanto do ponto de visto acadêmico como prático, mas necessário para eliminar falhas nos projetos. Galvão e Patah (2017), que realizaram estudo bibliométrico sobre a gestão de projetos sustentáveis, constataram que a literatura na área ainda é incipiente e demanda novos estudos. Decorre daí a relevância das 
pesquisas propondo metodologia de aprendizagem de equipes de projeto. Floriani e Steil (2021) demonstraram que a aprendizagem em equipe é fundamental para o desenvolvimento de projetos.

Paula (2008) enfatiza que um projeto de DSRL deve contemplar um planejamento de curto, médio e longo prazo. Ademais, deve envolver:

(i) parceria entre estado, mercado e sociedade;

(ii) protagonismo local e regional;

(iii) mobilização, convergência e integração dos recursos disponíveis (internos e externos);

(iv) capacitação permanente para a gestão local e regional;

(v) diversificação econômica;

(vi) integração das atividades econômicas em cadeias produtivas com maior competitividade;

(vii) sensibilização dos participantes;

(viii) organização de Fórum regional e local;

(ix) diagnóstico participativo local e regional;

(x) plano de desenvolvimento regional e local;

(xi) pacto de desenvolvimento regional e local; (xii) implementação da agenda regional e local.

Para Paula (2008), as principais condicionantes de sucesso de um projeto de DSRL são:

(i) adesão da comunidade regional e local;

(ii) apoio dos prefeitos e autoridades locais e regionais; (iii) apoio dos governos estadual e federal;

(iv) ampla rede de parcerias (ONGs, empresas, cooperação internacional);

(v) capacitação continuada ou permanente.

Quanto a execução de um projeto de DSRL, Paula (2008) afirma que a mesma deve ser acompanhada de um suporte governamental ou profissional especializado. Esse suporte deve envolver:

(i) identificação de vocações e oportunidades;

(ii) identificação de vantagens comparativas e competitivas;

(iii) (capacitação de empreendedores;

(iv) diversificação de negócios;

(v) integração de setores produtivos;

(vi) acesso ao crédito.

\subsubsection{Governança metropolitana sustentável}

O federalismo caracteriza-se pela associação das esferas de governo para melhorar a ação descentralizadora procurando, ao mesmo tempo, a unificação de objetivos e integração administrativa (Silva, 2009). Matus (2000) destaca a importância da "tecnopolítica" como instrumento capaz de sanar a baixa capacidade de governo, agindo diretamente sob os atores que atuam na direção política de governo. Para o autor, nos âmbitos dos objetivos federativos torna-se importante o papel da governança, ou seja, a capacidade de gestão, de técnica, de habilidades, de métodos e de recursos de administração. 
O fortalecimento do papel dos municípios tem um impacto político que se confronta com problemas de governança a nível local: a capacidade de governança e, particularmente, a capacidade técnica e institucional dos municípios. Em outro plano, questiona a autonomia financeira e a dificuldade de equilíbrio fiscal (Baldissera, 2015) dos entes locais. No Brasil, o aspecto histórico da centralização do governo e seu caráter autoritário (Baldissera, 2015) constitui um fator relevante na ação de governança e governabilidade.

De acordo com Cardoso e Cunha (2015) a nova realidade latino-americana, caracterizada pela baixa capacidade de governar, demanda uma mudança no processo de governança: (i) envolvimento e participação da comunidade local; (ii) capacitação política e técnica dos gestores e técnicos; (iii) profissionalização do servidor público; (iv) busca de cooperação a nível regional e global; (v) fortalecimento do controle público sobre o poder privado; (vi) planos estratégicos regionais ou metropolitanos.

Os planos a partir de uma visão estratégica de médio e longo prazo, sistematizam e aprimoram novas formas de governança em consonância com os desafios regionais e metropolitano.

\subsubsection{Integração social, econômica e ambiental} entre municípios para o desenvolvimento sustentável

Sobre a capacidade de se desenvolver considerando a sustentabilidade, Rodrigues e Santos (2018) afirmam que a transformação da terra, do trabalho e da organização produtiva em mercadorias implica em mudanças sociais e ambientais desastrosas pelo fato do homem, natureza e organização produtiva serem elementos que compõem um território. Portanto, são inseparáveis e não são originalmente produtos.

O sistema de mercado criado e estabelecido na sociedade contemporânea, em todos os seus aspectos, têm como objetivo a mercantilização do território, desconsiderando seus aspectos ambientais, econômicos e sociais (Rodrigues \& Santos, 2018). Para considerar a dinâmica territorial, nos modelos de desenvolvimento sustentável, é preciso reconhecer que cada comunidade, a partir de suas instituições específicas, constrói singularmente suas interações sociais, ambientais e econômicas e, consequentemente, seus modelos de desenvolvimento. Infelizmente, este modelo não se aplica na maioria das cidades e todo o ambiente e cultura das comunidades é ignorado para que seja seguido o padrão de desenvolvimento econômico.

Sobre a governança adaptativa para o DSRL, segundo Inácio, Rodrigues, Xavier, Wittmann \& Minussi (2013), esta objetiva a possibilidade de ligar indivíduos, organizações, agências e instituições em diversos níveis organizacionais. Os impactos ambientais, sociais e econômicos entre as cidades devem ser 
mapeados para que o DSRL ocorra, já que pessoas, recursos ambientais e riquezas interagem de forma a não respeitar as fronteiras delimitadas geograficamente, sendo extremamente importantes e estratégicos para o desenvolvimento local.

É válido lembrar que o DSRL está inserido dentro de uma sociedade capitalista, onde o lucro é o grande objetivo. Sendo assim, é preciso conciliar os três pilares básicos do desenvolvimento sustentável de uma forma diferenciada. Um exemplo citado por Rodrigues e Santos (2018), é a dimensão social para o DSRL, que considera a cultura como elemento fundamental no processo de desenvolvimento, uma vez que o desenvolvimento que se pretende sustentável, deve ter como prioridade aquilo que há de mais humano no trabalho, ou seja, o próprio homem e sua interação com o meio.

Nesse sentido, a integração social, econômica e ambiental entre municípios da RIDE-Plus Ultra poderá ser um importante componente do seu projeto de desenvolvimento.

\subsubsection{Cooperativismo e consórcio intermunicipal sustentável}

O desenvolvimento sustentável pressupõe o desenvolvimento capaz de suprir as necessidades da geração atual, sem comprometer a capacidade de atender as necessidades das gerações futuras, como preceituado no Relatório Nosso Futuro Comum - Relatório de Brundtland. É possível mapear formas diferentes de se desenvolver sustentavelmente. Mas, para isso, é necessário que haja planejamento, principalmente no uso dos recursos naturais finitos. Uma das alternativas para que esse planejamento seja efetivo é o cooperativismo (Pochmann, 2012).

O cooperativismo se apresenta como uma estrutura onde não há a posição de proprietário, mas sim de associados, isto é, um modelo que permite uma inclusão econômica, dado que não há uma hierarquia entre os participantes. Esse sistema se utiliza dos princípios de igualdade e democracia participativa, sendo também um modelo de inclusão social, que enriquece a discussão referente ao conceito de propriedade (Schneider, 2015).

Outro ponto do cooperativismo que pode contribuir para o desenvolvimento sustentável entre cidades é que o poder de decisão não está vinculado a posse. Ou seja, não importa o capital, em uma cooperativa todos os votos dos associados têm igual peso, mesmo dentro do contexto do cooperativismo entre cidades. O mesmo ocorre dentro de uma Região Integrada de Desenvolvimento, onde não importa qual cidade é maior, ou possui maior geração de riqueza, todas as cidades envolvidas têm o mesmo poder de decisão (Schneider, 2015).

Cooperativismo significa resolver problemas sociais por meio de comunidades de cooperação. O cooperativismo surgiu como uma alternativa entre o capitalismo e o socialismo. Este ideal tem seu berço na Inglaterra no século XIX (Cavallaro, Furlanetti \& Krajauer, 2016). 
Para Sandroni (2005, p. 190), cooperativa é "uma empresa formada e dirigida por uma associação de usuários, que se reúnem em igualdade de direitos com objetivo de desenvolver uma atividade econômica ou prestar serviços comuns, eliminando os intermediários".

As cooperativas podem assumir diferentes modalidades: de produção, de consumo, de crédito, de troca e comercialização, de venda por atacado ou de assistência médica. Existem cooperativas mistas que assumem diferentes atividades econômicas. As mais comuns, são cooperativas de produção, de crédito e de consumo (Sandroni, 2005).

Já os Consórcios Intermunicipais, nascem da iniciativa e do diálogo de gestores públicos e membros da sociedade com a finalidade de trazer benefícios para a região a partir de interesses em comum. Seu principal objetivo é a otimização na gestão pública municipal e regional através de uma maior sensibilidade política pela aproximação com a realidade dos cidadãos. Os mesmos podem ser utilizados em praticamente todas as áreas de atuação e de responsabilidade da administração pública, tais como: educação, turismo, cultura, meio ambiente, energia, urbanização, transporte e mobilidade, saneamento básico, saúde, dentre outras (Viana et al., 2008).

Os Consórcios Intermunicipais são relevantes tanto no atendimento à população, por meio da viabilidade da prestação de serviços públicos, como para a própria gestão pública, que isoladamente não conseguiria atingir seus objetivos. Assim sendo, os Consórcios Intermunicipais se constituem numa parceria entre as várias prefeituras, aumentando suas capacidades de solucionar problemas comuns, porém sem lhes retirar a autonomia (Guimarães, 2010).

No caso da RIDE-Plus Ultra, os interesses comuns seriam, pelos menos em tese, distribuídos de forma inteligente e eficazmente mais barata pela compra licitatória em grande número, valendo-se da organicidade dos municípios, em consórcio, para execução proveitosa dos serviços públicos, antes em desarmonia e perdulária. Neste sentido, tanto as cooperativas quanto os consórcios intermunicipais, poderão ser importantes componentes do Projeto de desenvolvimento sustentável da RIDE-Plus Ultra.

\subsubsection{Políticas públicas para o desenvolvimento local sustentável}

O planejamento é um esforço para otimizar as relações entre meios e fins, com a racionalização das ações na realidade regional ou urbana para melhor aplicar os recursos disponíveis e obter resultados com mais eficiência. A gestão é o processo no qual a sociedade e os agentes sociais são consultadas ativamente na orientação do seu destino e do seu ambiente natural. A gestão pública está ligada à participação da população nas escolhas sociais (Fernandez, 2014). 
Os novos princípios de planejamento e gestão afirmam que agentes sociais devem ser consultados em análises e debates a fim de definir prioridades na execução das propostas. A gestão social articula agentes sociais e instituições para conciliar interesses e obter acordos para o planejamento do desenvolvimento das cidades. Existem diversos mecanismos para garantir a participação dos cidadãos no Poder Público, como a iniciativa popular, o plebiscito, consultas e conselhos. O planejamento necessita ser participativo e democrático e ter o debate de todos os agentes sociais, ou seja, a opinião das comunidades é fundamental. É importante destacar que a sustentabilidade busca o manejo correto dos recursos naturais, o desenvolvimento socialmente justo e economicamente viável (Fernandez, 2014). Assim, entende-se que a concretização do projeto de desenvolvimento sustentável da RIDE-Plus Ultra envolve, necessariamente, a participação de cidadãos e comunidades.

\subsubsection{Aglomerações produtivas, clusters, APLs e incubadoras}

As empresas são agentes que otimizam os lucros e possuem decisões racionais para satisfazer suas necessidades. A cooperação entre empresas é analisada com racionalidade econômica, uma forma encontrada por um ator para minimizar custos de transações que vêm quando ele atua isoladamente e sem cooperação, num ambiente dinâmico e imprevisível. As empresas buscam minimizar os custos de transação e estabelecem uma fronteira ótima entre as atividades que controlam e aquelas que são feitas por transações no mercado (Vale \& Castro, 2010).

A localização espacial da produção das empresas atraiu, desde cedo, a atenção dos economistas. A aglomeração produtiva competitiva é um espaço territorial onde atores buscam interação e cooperação, visando reduzir custos de transação e gerar uma dinâmica territorial própria. Surgem os conceitos de sistemas produtivos e inovativos locais (SPIL) e de arranjos produtivos locais (APL). Os SPIL são agentes econômicos, políticos e sociais que estão no mesmo território e desenvolvem atividades econômicas parecidas e vínculos expressivos de produção, cooperação e aprendizado (Vale \& Castro, 2010). Entretanto, de acordo com Teixeira e Teixeira (2011), há necessidade de delimitar esses conceitos. Observa os pesquisadores que no Brasil os APLs são apresentados como possuidores de maiores valores de capital social e perspectiva de desenvolvimento local. Os Clusters, por sua vez, acentuam a visão de concentração na obtenção de maiores ganhos econômicos. Estas formas organizacionais, no entanto, podem coexistir.

Os APLs e Clusters podem ser classificados também como aglomerados produtivos capazes de promover a competitividade e o desenvolvimento de um território. Sua configuração produtiva-territorial 
estão caracterizadas: a) por uma "cadeia produtiva" de fluxos de bens, serviços e informações, priorizando um produto ou um ramo de produção; b) por um "sistema produtivo" que envolve unidades de produção, serviços e fornecedores, complementados por outros agentes, lideranças locais e instituições públicas e privadas (Alvarenga et al., 2013).

Outro meio de se estimular o desenvolvimento local é através das incubadoras de empresas que são locais onde nascem, crescem e desenvolvem-se pequenos negócios, na maioria dos casos de âmbito tecnológico. Nesse local, os negócios são amparados por uma infraestrutura comum e, por vezes, com a presença de uma Universidade, de forma a incentivar a criação e transformação de ideias em produtos, serviços e processos (Barbosa \& Hoffmann, 2013). São também formas de unir tecnologia, capital e know how para alavancar o talento empreendedor $\mathrm{e}$ acelerar o desenvolvimento de novas empresas (Costa et al., 2007).

Segundo Mantovani, Granito, Cabral \& Leite (2006) as incubadoras possuem quatro tipos diferentes possíveis:

- Incubadoras sem fins lucrativos: são criadas por câmaras de comércio ou associações (industriais ou comunitárias). Seu objetivo principal é proporcionar desenvolvimento econômico para uma pequena região ou setor industrial específico;
- Incubadora de Universidades: transformam, incentivam, financiam conhecimentos desenvolvidos dentro das Universidades. Seja através da disseminação de novas teorias ou criação de novos produtos e tecnologias;

- Incubadoras Privadas: tem como objetivo o lucro. Nesse contexto, as incubadoras privadas também buscam a criação de um ambiente empreendedor que priorize o desenvolvimento de uma região ou comunidade;

- Incubadora Pública: sua função é a geração de empregos, desenvolvimento de novos negócios e produtos que gerem uma diversificação econômica e estimule o empreendedorismo.

As incubadoras, segundo Mantovani, Granito, Cabral \& Leite (2006), têm um papel de suma importância para o desenvolvimento local. Os autores afirmam que quando uma incubadora se propõe a realizar um desenvolvimento local existe a possibilidade de gerar impactos positivos na sociedade a partir de:

- Aumento da autonomia local e instituição de novos mecanismos para tomada de decisão;

- Aumento da capacidade local de geração de renda e excedentes. É importante realocar parte do excedente diretamente para incentivo ao 
desenvolvimento;

- Foco no ponto de inclusão social;

- Envolvimento e conscientizar da população quanto a importância de um desenvolvimento que respeita os recursos naturais. Criação de responsabilidade ambiental visando uma racionalização e maior preservação dos recursos locais.

\subsubsection{Soluções propostas e ações de curto, médio e longo prazo}

Após desenvolver as pesquisas bibliográficas, consultar especialistas na área de políticas públicas para desenvolvimento regional e local e realizar um estudo de campo para fundamentar as suas propostas, os alunos apresentam soluções, em caráter de propostas, considerando a seguinte perspectiva temporal: curto, médio e longo prazo. Foi determinado que o curto prazo abrangeria o período de 01 a 12 meses, o médio prazo de 13 meses a 24 meses e o período de longo prazo remeteria a além dos 25 meses. No entanto, esta classificação não tem caráter definitivo, pois de acordo com a conjuntura e os fatores sociopolíticos e econômicos, estes períodos temporais podem ser alterados.

\section{prazo foram:}

\section{As soluções apresentadas para o curto}

- Envolver a participação da comunidade, em diversos meios e modos, com o objetivo ouvir e identificar as prioridades públicas da sociedade para a RIDE e, com isto, integrar a população e a sociedade civil como participantes do processo de desenvolvimento;

- Promover reuniões periódicas dos Prefeitos para avaliar e planejar as ações prioritárias, constituindo, como suporte, equipes temáticas de aprofundamento das questões centrais da RIDE;

- Criar uma Escola de Gestão Estratégica para promover a capacitação técnica e política dos servidores das diversas esferas dos governos locais, objetivando aprimorar a gestão administrativa, a elaboração de projetos e a captação de recursos;

- Pesquisar sobre as atividades econômicas e empresariais do território nas últimas décadas, objetivando identificar sua "vocação produtiva", suas forças e deficiências, possibilitando a elaboração de um Projeto preliminar de desenvolvimento econômico sustentável do território;

- Criar um Fórum do Empreendedorismo Social visando envolver a população dos bairros mais pobres e periféricos, com o objetivo de motivar e conhecer as experiências práticas das diversas modalidades de economia solidária, 
cooperativas e associações.

\section{As propostas de soluções para o médio} prazo foram:

- Reuniões temáticas dos técnicos dos governos locais para compartilhamento de estratégias e dos planos de ação nas atividades comum específicas;

- Curso de gestão estratégica e administrativa de Consórcios Intermunicipais Sustentáveis direcionadas aos agentes públicos e administrativos, objetivando a formação tecnopolítica para gestão dos consórcios da RIDE;

- Promover o Fórum de Desenvolvimento Sustentável da RIDE com a participação dos empresários e da sociedade, objetivando a elaboração do projeto estratégico de desenvolvimento econômico;

- Disponibilizar cursos de formação e capacitação para os líderes populares, objetivando a administração e gestão de empreendimentos sociais e comunitários.

\section{As propostas de soluções para o longo} prazo foram:

- Revisão dos planos estratégicos e de cooperação entre os municípios da
RIDE com objetivo de analisar os resultados obtidos, aprimorar as ações e delinear as estratégias e os passos seguintes;

- Apresentação do plano de Consórcio Intermunicipal Sustentável à sociedade, objetivando tornar pública as mudanças administrativas decorrentes;

- Criação de Incubadora de Empresas e do Polo Tecnológico da RIDE com o objetivo de acelerar a criação de pequenas e médias empresas com perfil tecnológico capazes de impulsionar a cadeia produtiva do território e promover sua competividade;

- Criação de incubadora para os empreendimentos da economia solidária e cooperativas, objetivando garantir o fomento, o suporte logístico e a assessoria necessária ao desenvolvimento local nas comunidades.

Os recursos financeiros, administrativos e humanos para realizações dos eventos e dos projetos devem ser disponibilizados proporcionalmente pelos municípios integrantes da RIDE-Plus Ultra. Recursos dos governos estaduais e federal, obtidos através de projetos devem ser destinados às finalidades específicas. Fundamentais são as parcerias com as empresas da região, comprometendo-as, por meio da sua participação, com o projeto de desenvolvimento 
sustentável. A realização dos eventos e ações deve contar, ainda, com a participação e a assessoria das Universidades e Instituições de Ensino da RIDE e fora dela, além dos órgãos patronais e dos trabalhadores.

\subsection{Apresentação}

A apresentação dos resultados dos trabalhos dos alunos foi conduzida tendo, como referência, o conteúdo do relatório final do PBL. Com a apresentação oral, os alunos relacionaram a questão norteadora do problema com a contextualização teórica construída, a fim de comunicar as soluções propostas para o projeto de desenvolvimento sustentável da RIDE-Plus Ultra. A troca de experiências entre os alunos foi fomentada em três etapas. Primeiramente apresentaram a contextualização do problema considerando os fatos e dados depreendidos do texto. Em seguida, foram apresentados os sete conceitos selecionados como relevantes para a construção de soluções para o PBL. E por fim, os alunos discorreram sobre as soluções para a promoção do desenvolvimento sustentável regional e local, a partir do planejamento de curto, médio e longo prazo para a RIDE-Plus Ultra.

\subsection{Debate}

$\mathrm{O}$ debate entre os membros da equipe ocorreu logo após a apresentação dos conceitos teóricos e das propostas de soluções. Essa prática, mediada pelo professor, permite aos alunos exercitar as suas habilidades comunicativas, favorece a aprendizagem e possibilita reforçar os conhecimentos prévios. Com a mediação do professor, os alunos formularam questões a respeito da apresentação, discutiram sobre as soluções de curto, médio e longo prazo e dialogaram sobre os conteúdos do projeto de desenvolvimento sustentável da RIDE-Plus Ultra. O debate foi rico em ideias, contribuiu para um melhor entendimento sobre o fenômeno estudado e permitiu estabelecer um consenso sobre a participação efetiva dos alunos na elaboração de uma proposta factível para a RIDE-Plus Ultra.

\subsection{Fundamentação teórica do professor}

A fundamentação teórica foi elaborada a partir de conteúdos teóricos relacionados com o estudo, os tópicos pesquisados e as soluções discutidas e debatidas entre e com os alunos. Essa é uma das etapas da aprendizagem ativa. No caso estudado foram evidenciados os caminhos para que o projeto de desenvolvimento sustentável da RIDE-Plus Ultra possa envolver a participação da comunidade. Como evidenciado pela equipe, com a elaboração do planejamento participativo, os cidadãos podem identificar as prioridades públicas da sociedade para a RIDE-Plus Ultra, em direção ao desenvolvimento socialmente justo e economicamente viável, como já apontado por Fernandez (2014). 


\section{Considerações finais}

A pesquisa demonstrou que é possível por meio de uma atividade em sala de aula e, utilizando o método PBL, encontrar soluções para implantar um projeto de desenvolvimento que vise solucionar os problemas econômicos, sociais e ambientais vivenciados por Regiões Integradas de Desenvolvimento.

Por meio do método PBL os estudantes foram estimulados a pesquisar, pensar criticamente e propor solução para o problema do desenvolvimento regional. As propostas apontaram que a solução do problema exige visão holística e requer o envolvimento do governo, a participação da iniciativa privada e da comunidade como um todo. E ainda, que o desenvolvimento é um processo e requer iniciativas em diferentes horizontes temporais.

Especificamente, após desenvolver as pesquisas bibliográficas, consultar especialistas na área de políticas públicas para desenvolvimento regional e local e realizar um estudo de campo para fundamentar as suas propostas, os alunos apresentaram soluções, em caráter de propostas, considerando a perspectiva temporal de curto, médio e longo prazo.

A forma de ensinar e aprender tem evoluído e o PBL permite conduzir os alunos para uma nova realidade, onde devem ser mais ativos e protagonistas do seu próprio desenvolvimento. Pelos resultados apresentados, é possível afirmar que a metodologia PBL contribuiu para o aprofundamento quanto às estratégias públicas e privadas aplicadas nos territórios visando o desenvolvimento sustentável, permitindo o estudo alcançar o seu objetivo principal.

O problema hipotético sobre a RIDE-Plus Ultra evidencia a indispensável integração de toda a sociedade, que deve ser envolvida desde os primeiros passos, para que o desenvolvimento sustentável realmente aconteça. Ações de pesquisa, de formação e capacitação tecnopolítica dos atores sociais e o público, também são fundamentais para fundamentar a implementação das ações e obras voltadas ao desenvolvimento sustentável do território. Os planos pensados e apresentados considerando curto, médio e longo prazo, são estratégicos para que as decisões sejam tomadas de forma consciente e dentro do cenário real do território, acompanhando o tempo de evolução daquela sociedade.

Assim, este estudo propõe que as realizações das etapas apresentadas permitem a evolução da RIDE, considerando o desenvolvimento sustentável, podendo alcançar o objetivo de se tornar uma Região Metropolitana. Este trabalho pode servir como base para estudantes, professores e demais interessados, no mesmo objetivo comum.

\section{Referências}

Albagli, S. (2006). Conhecimento, inclusão social e desenvolvimento local. Inclusão Social, 1(2), 17-22.

Albarello, C. B., Albarello, L., \& Siedenberg, D. 
R. (2006). Estratégias capazes de contribuir no processo de promoção do desenvolvimento sustentável na esfera local e regional. Revista de Administração, 5(8), 39-63.

Alvarenga, R. A. M., Matos, F. R. N., Machado, D. Q., Sobreira, M. C., \& Matos, L. B. S. (2013). Arranjo produtivo local e desenvolvimento sustentável: uma relação sinérgica no município de Marco (CE). Revista de Administração Mackenzie, 14(5), 15-43.

Araújo, A. M. P., Fregonesi, M. S. F. A., Soares, M. A., \& Slomski, V. G. (2010). Aplicação do Método Problem-based Learning (PBL) no de Curso de Especialização em Controladoria e Finanças. Recuperado em 13 junho 2019, de https://repositorio.usp.br/item/002153511

Baldissera, D. S. (2015). Consórcios Públicos Intermunicipais no Brasil. Dissertação de Mestrado, Fundação Getúlio Vargas, Rio de Janeiro, RJ, Brasil.

Barbosa, L. G. F., \& Hoffmann, V. E. (2013). Incubadora de empresas de base tecnológica: percepção dos empresários quanto aos apoios recebidos. Innovation and Management Review, 10(3), 208-229.

Boevé, A. J., Meijer, R. R., Bosker, R. J., Vugteveen, J., Hoekstra, R., \& Albers, C. J. (2017). Implementing the Flipped Classroom: An Exploration of Study Behaviour and Student Performance. Higher Education, 74(6), 1015-1032.

Borges, M. C., Chachá, S. G. F., Quintana, S. M., Freitas, L. C. C., \& Rodrigues, M. L. V. (2014). Aprendizado Baseado em Problemas. Medicina, 47(3), 301-307.

Borochovicius, E., \& Tortella, J. C. B. (2014). Aprendizagem Baseada em Problemas: um método de ensino-aprendizagem e suas práticas educativas. Ensaio: Avaliação e Políticas Públicas em Educação, 22(83), 263294.
Cardoso, J. C., \& Cunha, A. S. (Orgs). (2015). Planejamento e avaliação de políticas públicas. Brasília: Ipea.

Carvalho, K. C. (2018). Aprendizagem Baseada em Problemas. Campinas: PUC-Campinas. (Palestra apresentada em 08 de fevereiro de 2018).

Castaneda-Ayarza, J. A., De Benedicto, S. C., Ferreira, D. H. L., \& Barbosa, M. (2019). PBL method in the formative process in postgraduate courses. International Journal for Innovation Education and Research, 7(12), 333-347.

Cavalcanti, C. T. A., \& Silva, I. R. M. (2016). Contribuições e desafios da sustentabilidade na gestão de projetos. Revista de Gestão e Projetos-GeP, 7(3), 20-28.

Cavallaro, G. M., Furlanetti, E. W., \& Krakauer, P. V. C. (2016). Cluster e desenvolvimento local: o caso da Cooperativa Veiling Holambra. Espacios, 37(19), 11-22.

Christopoulos, T. P., \& Steinbeck, R. (2016). Institutionalising the learning process through innovation and participative practices. International Journal Innovation and Learning, 19(2), 188-205.

Costa, C. V., Silva, L. B., Penha, R. H. S., Silva, A. N., Elias, R. S., Pereira, V. S., \& Teixeira, M. G. C. (2007, setembro). Incubadora de Empresa de Base Tecnológica. Recuperado em 21 maio 2021, de http://www.anpad.org.br/diversos/down_zips/ 33/GCT-B2278.pdf

ENAP - Escola Nacional de Administração Pública. (2018). Políticas públicas e governo local. Módulo 3 (Desenvolvimento local e sustentabilidade). Brasília: ENAP.

Escrivão Filho, E., \& Ribeiro, L. R. C. (2008). Inovando no ensino de administração: uma experiência com a Aprendizagem Baseada em 
Problemas (PBL). Cadernos EBAPE. BR, 6, $1-9$.

Feil, A. A., \& Schreiber, D. (2017).

Sustentabilidade e desenvolvimento sustentável: desvendando as sobreposições e alcances de seus significados. Cadernos EBAPE.BR, 15(3), 667-681.

Fernandez, F. N. (2014. Planejamento regional e o desafio da sustentabilidade. Revista Política e Planejamento Regional, 1(1), 81-102.

Floriani, E. V., \& Steil, A. V. (2021). Processos de aprendizagem em equipe de projeto que utiliza metodologia ágil. Revista de Gestão e Projetos - GeP, 12(1), 149-171.

Fortes, G. B., \& Moraes, F. (2016). Federalismo e democracia. RIL - Revista de Informação Legislativa, 53(211), 199-226.

Freitas, R. A. M. M. (2012). Ensino por problemas: uma abordagem para o desenvolvimento do aluno. Educação e Pesquisa, 38(2), 403-418.

Frezatti, F., Martins, D. B., Mucci, D. M., \& Lopes, P. A. (2018). Aprendizagem Baseada em Problemas (PBL). São Paulo: Atlas.

Galvão, G. D. A., \& Patah, L. A. (2017). Gestão de projetos sustentáveis e inovadores: um estudo bibliométrico. Revista de Gestão e Projetos-GeP, 8(30, 29-49.

Gholami, M., Moghadam, P. K., Mohammadipoor, F., Tarahi, M. J., Toulabi, T., \& Pour, A. H. H. (2016). Comparing the effects of problem-based learning and the traditional lecture method on critical thinking skills and metacognitive awareness in nursing students. Nurse Education Today, 45, 16-21.

Goldenberg, J. (2015). Energia e sustentabilidade. Revista Cultura e Extensão USP, 14, 33-43. DOI:

http://dx.doi.org/10.11606/issn.23169060.v14i0p33-43
Guimarães, T. C. (2010). O consórcio público como instrumento de fortalecimento do federalismo brasileiro. Dissertação de Mestrado, Fundação João Pinheiro, Belo Horizonte, MG, Brasil.

Inácio, R. de O., Rodrigues, M. D., Xavier, T. R., Wittmann, M. L., \& Minussi, T. N. (2013). Desenvolvimento regional sustentável: abordagens para um novo paradigma. Desenvolvimento em Questão, 11(24), 6-40.

Japiassú, C. E., \& Guerra, I. F. (2017). 30 anos do Relatório Brundtland. Revista de Direito da Cidade, 09(4). 1884-1901.

Lara, L. G. A., \& Oliveira, S. A. (2017). The ideology of economic growth and the business discourse of sustainable development. Caderno EBAPE.BR, 15(2), 326-348.

Leon, L. B., \& Onófrio, F. Q. (2015). Aprendizagem baseada em problemas na graduação médica - uma revisão da literatura atual. Revista Brasileira de Educação Médica, 39(4), 614-619.

Lima, A. R. M. (2006). Desenvolvimento local integrado e sustentável - DLIS. Dissertação de Mestrado, Universidade Federal da Paraíba, João Pessoa, PB, Brasil.

Machado, D. Q., \& Matos, F. R. N. (2020). Reflexões sobre desenvolvimento sustentável e sustentabilidade. Revista de Administração, Contabilidade e Sustentabilidade, 10(3), 1426.

Marques, A. C. C., Barazzutti, A., Senna, A. J. T., \& Alves, R. R. (2014). Análise das estratégias para a promoção do desenvolvimento local. Revista Estudo \& Debate, 21(1), 200-216.

Matus, C. (2000). O Líder sem Estado-Maior. São Paulo: Fundap. 
Nagarajan, S., \& Overton, T. (2019). Promoting systems thinking using project and problem based learning. Journal of Chemical Education, 96(12), 2901-2909.

Nascimento, E. P. (2012). Trajetória da sustentabilidade: do ambiental ao social, do social ao econômico. Estudos Avançados, 26(74), 51-64.

Mantovani, D. M. N., Granito, R. A. N., Cabral, D. G., \& Leite, M. F. B. (2006). O papel das incubadoras de empresas no desenvolvimento local: um estudo de caso. RAI - Revista de Administração e Inovação, 3(1), 90-101.

Nista, N.A., Jannuzzi, C.A.S.C., Falsarella, O.M., \& De Benedicto, S.C. (2020). Society and sustainable development: Animal rights in sustainability discourse. Ambiente \& Sociedade, 23, 1-18.

Oliveira, M. L. (2018). Desenvolvimento sustentável e os municípios. Revista de Direito e Sustentabilidade, 4(1), 59-76.

Paula, J. (2008). Desenvolvimento local: como fazer? Brasília: SEBRAE.

Pochmann, M. (2012). Repartir para o consumo sustentável. Recuperado em 09 março 2019, de

http://www.ihu.unisinos.br/noticias/507747repartirparaoconsumosustentavel

Pott, C. M., \& Estrela, C. C. (2017). Histórico ambiental: desastres ambientais e o despertar de um novo pensamento. Estudos Avançados, 31(89), 271-283

Ribeiro, L. R. C. (2010). Aprendizagem baseada em problemas: Uma experiência no ensino superior. São Carlos: EdUFSCar.

Ribeiro, L. R. C., \& Mizukami, M. G. N. (2005). Problem-based learning: a student evaluation of implementation in postgraduate engineering education. European Journal of Engineering Education, 30(1), 137-149.
Rodrigues, W., \& Santos, N. S. (2018). Desenvolvimento territorial no Brasil: uma análise a partir da concepção teórica de Karl Polanyi. Interações, 19(1), 119-135.

Sachs, I. (2009). Caminhos para o desenvolvimento sustentável. Rio de Janeiro: Garamond.

Sachs, J. (2015). The Age of Sustainable Development. New York, NY: Columbia University Press.

Sandroni, P. (2005). Dicionário de Economia do século XXI. Rio de Janeiro: Record.

Sartori, S., Latrônico, F., \& Campos, L. M. S. (2014). Sustentabilidade e desenvolvimento sustentável: uma taxonomia no campo da literatura. Ambiente \& Sociedade, 17(1), 0122.

Schneider, J. O. (2015). Cooperativismo e desenvolvimento sustentável. Otra Economía, 9(16), 94-104.

Silva, J. A. (2009). Comentário Contextual à Constituição ( $6^{\circ}$ ed.). São Paulo: Malheiros.

Silva, I. M., Araújo, M. L. F., Leão, M. B. C., \& Lins, W. C. B. (2017). Percepções de Estudantes de um Programa de PósGraduação sobre a Aprendizagem Baseada em Problemas. Enseñanza de las ciencias, n. $^{\circ}$ Extra, 743-748.

Souza, S. C., \& Dourado, L. (2015). Aprendizagem Baseada em Problemas (ABP): um método de aprendizagem inovador para o ensino educativo. Holos, 31(5), 182-200.

Sugahara, C. R., Jannuzzi, C. A. S. C., \& Sousa, J. E. (2012). O ensino-aprendizagem baseado em problema e estudo de caso num curso presencial de Administração - Brasil. Revista Iberoamericana de Educación, 60(1), 1-9.

Tainter, C. R., Wong, N. L., Cudemus-Deseda, 
G. A., \& Bittner, E. A. (2017). The "flipped classroom" model for teaching in the intensive care unit. Journal of Intensive Care Medicine, 32(3), 187-196.

Teixeira, M. C., \& Teixeira, R. M. (2011). Relacionamento, cooperação e governança em arranjos produtivos locais. REAd. Revista Eletrônica de Administração, 17(1), 237-269.

Vale, G. M. V., \& Castro, J. M. (2010). Clusters, arranjos produtivos locais, distritos industriais: reflexões sobre aglomerações produtivas. Análise Econômica, 28(53), 8197.

Valente, J. A., Almeida, M. E. B., \& Geraldini, A. F. S. (2017). Metodologias ativas: das concepções às práticas em distintos níveis de ensino. Revista Diálogo Educacional, 17(52), 455-478.

Viana, A. L. A., Ibañez, N., Elias, P. E. M., Lima, L. D., Albuquerque, M. V, \& Iozzi, F. L. (2008). Novas perspectivas para regionalização da saúde. São Paulo em Perspectiva, 22(1), 92-106.

Ylitalo, M., Jääskeläinen, M., Horelli, J., \& Väänänen, M. (2012). R\&D with PBL in engineering education. International Journal Innovation and Learning, 12(1), 85-94. 